\title{
The dependence of the "experimental" pion-nucleon sigma term on higher partial waves
}

\author{
J. Stahov \\ Abilene Christian University, Abilene, TX, 76999, USA \\ and \\ University Tuzla, 35000 Tuzla, Bosnia and Herzegovina
}

December 24, 2018

\begin{abstract}
A dependence of the value of the $\pi N$ sigma term on higher partial waves is discussed. Two recent predictions of a high value of sigma term are scrutinized. It has been concluded that the main reason for obtaining high values of the sigma term are input $\mathrm{D}$ waves that are not consistent with analyticity
\end{abstract}

\section{Introduction}

The value of the $\pi N$ sigma term $\Sigma$ is given in terms of the $\bar{D}^{+}$amplitude (bar indicates that the pseudovector Born term is subtracted) at the Chang-Dashen (CD) point $\nu=0, t=2 m_{\pi}^{2}$ :

$$
\Sigma=F_{\pi}^{2} \bar{D}^{+}\left(\nu=0, t=2 m_{\pi}^{2}\right),
$$

where $F_{\pi}=92.4 \mathrm{MeV}$ is the pion decay constant. For details concerning the $\pi N$ kinematics we refer to reference|1]. Generally, there are two kinds of methods used to calculate the $\bar{D}^{+}$amplitude at the CD point. The first method uses dispersion relations to calculate $\bar{D}^{+}\left(0,2 m_{\pi}^{2}\right)$ directly. The second method determines the coefficients in the subthreshold expansion of the $\bar{D}^{+}$amplitude:

$$
\begin{aligned}
\bar{D}^{+}\left(0,2 m_{\pi}^{2}\right) & =\left(\bar{d}_{00}^{+}+\bar{d}_{01}^{+} t+\bar{d}_{02}^{+} t^{2}+. .\right), \\
\Sigma & =\left(\bar{d}_{00}^{+}+\bar{d}_{01}^{+} t+\bar{d}_{02}^{+} t^{2}+. .\right) F_{\pi}^{2}, \\
\Sigma & \equiv \Sigma_{D}+\Delta_{R},
\end{aligned}
$$

where $\Sigma_{D}$ denotes contributions from the first two terms, and $\Delta_{R}$ is so called curvature term that includes contributions of quadratic and higher terms. Obtained values for $\Sigma$ range from $60 \mathrm{MeV}$ to $93 \mathrm{MeV}$.

It is of interest to understand which partial waves give important contributions to $\bar{D}^{+}$in each of the above mentioned methods. It is clear that the leading 
contributions come from the input $\mathrm{S}$ and $\mathrm{P}$ waves, but earlier evaluations(see reference[1] and references therein) show that contributions from D and higher partial waves must not be neglected.

\section{The role of the higher partial waves}

In order to demonstrate the importance of the higher partial waves in determination of the $\Sigma$ term, let's briefly describe two methods that have produced dramatically different results in the past few years. Gasser, Leutwyler, Locher, and Sainio $(G L L S)\left[3\right.$ proposed a method to improve results for $\Sigma_{D}$ previously derived from the KH80 [2] solution by taking into account newer, mutually consistent meson factory data below pion lab. momentum $k_{0}=185 \mathrm{MeV} / \mathrm{c}$. The method is based on six forward dispersion relations for the invariant amplitudes $D^{ \pm}, B^{ \pm}, E^{ \pm} \equiv \frac{\partial}{\partial t} D^{ \pm}$. D waves and higher partial waves are needed as a part of the input below a cutoff momentum $k_{0}$. Above $k_{0}$, results from one of the existing PW solutions are used. As a result, GLLS machinery predicts coefficients $\bar{d}_{00}^{+}$and $\bar{d}_{01}^{+}$in the subthreshold expansion. The curvature term $\Delta_{R}$ was determined using another method[ $[1$. Several further updates were made by Sainio. In reference [5 a value $\Sigma=62 \mathrm{MeV}$ was reported. Higher partial waves below cutoff momentum $k_{0}$ were taken from Ka85 solution[6]. It was pointed out that "results are rather insensitive to the choice of PW solution above the cutoff momentum". The most recent update was given in reference[7]. Results from Sp00 solution [8], including $D$ waves below $k_{0}$, were used. Obtained value, $\Sigma=93 \mathrm{MeV}$, is more than $50 \%$ higher than previously reported values. One concludes that GLLS machinery is sensitive to the input for D waves and higher partial waves below the cutoff momentum.

Starting from the fixed- $t$ dispersion relations for $t=2 m_{\pi}^{2}$, Olsson $[9]$ derived a sum rule in which the value of the $\bar{D}^{+}$amplitude at the CD point is expressed in terms of the S, P, D and higher partial waves threshold parameters. Using Koch's $[6]$ values for the $\mathrm{D}$ and higher partial wave scattering lengths, Olsson obtained value $\Sigma=(71 \pm 9) M e V$. Using $D$-and higher partial wave scattering lengths from VPI/GW solution Sm01[8], Olsson and Kaufmann[10 recently obtained significantly higher values ranging from $80 \mathrm{MeV}$ to $88 \mathrm{MeV}$.

Common to both of these high evaluations of the sigma term was the use of the higher partial waves at low energy from the latest VPI/GW solutions.

The dependence of the sigma term on the higher partial waves can be easily seen when applying Olsson sum rule. D-wave scattering lengths from the VPI/GW solution Sp00 lead to the sigma term value that is roughly $19 \mathrm{MeV}$ higher than value in reference [9] where D waves from Ka84 solution have been used. The VPI/GW group recently made some progress toward the lower values of the sigma term. D waves in their solutions Sm01 and Sm02 are smaller but still much higher than those from Ka84 solution. In Olsson sum rule contribution of the Sm02 D waves to the $\bar{D}^{+}$amplitude at CD point is $0.13 \mathrm{MeV}^{-1}$ ( compared to $-0.05 \mathrm{MeV}^{-1}$ from Ka84), that makes the sigma term roughly 11 $\mathrm{MeV}$ higher than in reference [9]. 


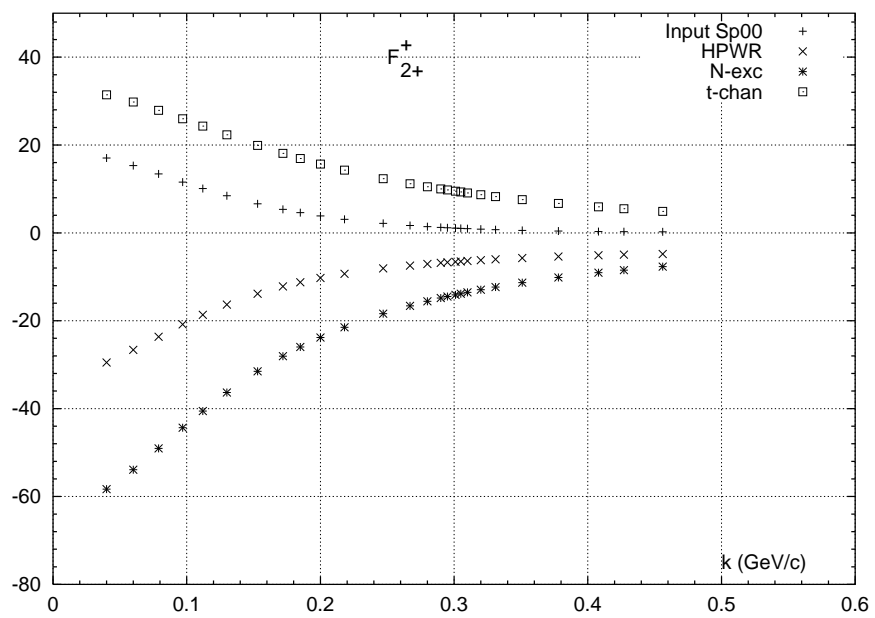

Figure 1: Comparizon of $\mathrm{F}_{2+}^{+}$from HPWR to input from Sp00.

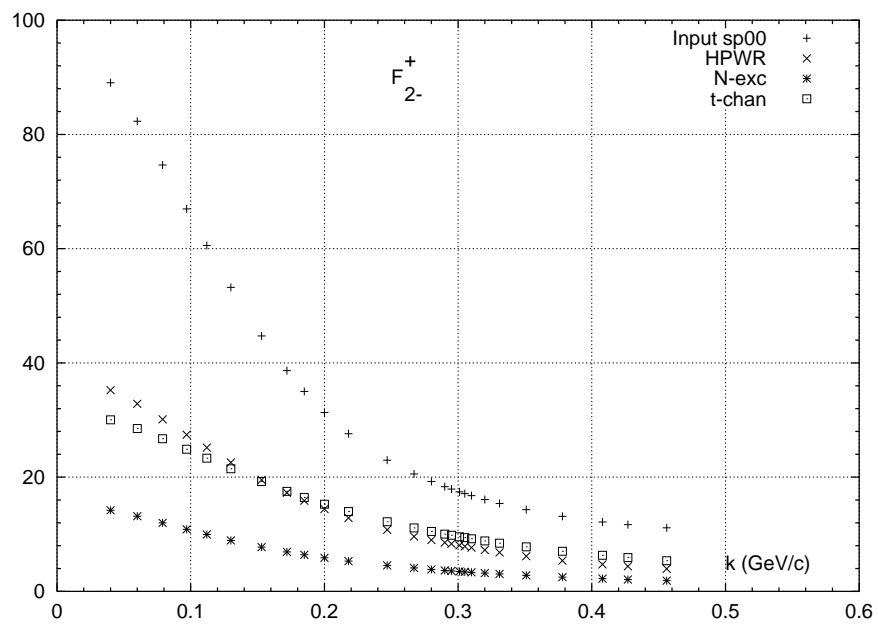

Figure 2: Comparizon of $\mathrm{F}_{2-}^{+}$from HPWR to input from Sp00. 
It is important to point out that below the GLLS cutoff momentum $k_{0}=185$ $\mathrm{MeV} / \mathrm{c}$ reliable values for $\mathrm{D}$ and higher partial waves can not be obtained from experimental data only. In another words, increase of the value of the sigma term due to contributions of $\mathrm{D}$ waves has no firm experimental foundation. D waves from VPI/GW solutions should be checked. One has to start from the first principle in $\pi N$ physics-Mandelstam analyticity and the analytic structure of the $\pi N$ partial waves. Consistency of a given partial wave with analyticity can be tested using one of the methods developed in the past (see reference 11 and references therein).

\section{Hyperbolic partial wave relations as a test of higher partial waves}

In the hyperbolic partial wave relations $(H P W R)$ [12], a given $s$-channel partial wave is expressed in terms of other $s$-channel partial waves and the $t$-channel partial waves, multiplied by corresponding $s$-channel and $t$-channel kernels. Explicitly known kernels in the HPWR reproduce the analytic structure of the $\pi N$ partial waves. In addition, there is also a contribution from a nucleon exchange term that is explicitly known as well. The method is superior compared to other methods when predicting higher partial waves (D waves and higher) in the low energy region $(k \leq 500 \mathrm{MeV} / \mathrm{c})$. In that case, there are two leading contributions, the nucleon exchange and the $t$-channel contribution 13. Due to behavior of the $t$-channel kernels, contributions from higher values of $t$ are strongly suppressed so that input available today makes it possible to obtain reliable predictions for the higher $\pi N$ partial waves. For example, the main contributions to the isospin even combinations of D waves, $F_{2 \pm}^{+}$, come from the kinematical region $t \leq 25 m_{\pi}^{2}$. Recent calculation 14. shows that our input from the $t$-channel in that kinematical region is fairly well known. Results from $H P W R$ for the isospin even combinations of reduced D waves $\left(F_{l \pm}^{+}=T_{l \pm}^{+} / q^{l+1}\right.$ , see ref.[1]) are shown in Fig. 1 and Fig. 2. It is evident that D waves from VPI/GW solutions Sp00 and Sm02 are not consistent with analyticity at low energies. For instance, the isospin even combination $\mathrm{F}_{2+}^{+}$(shown in Fig. 1) has the wrong sign. D waves from Sm02 solution are smaller but still far from being consistent with analyticity. D waves from the Sm02 solution are compared to HPWR calculations in Figures 3 and 4.

It is hard to understand the fact that VPI/GW group 15] constraints their $\mathrm{P}$ waves to the approximate Chew Low theory (16) (lowering their value of the sigma term for $6 \mathrm{MeV}$ ), completely ignoring at the same time results for $\mathrm{D}$ waves in Ka84 that are constrained by exact PWDR. 


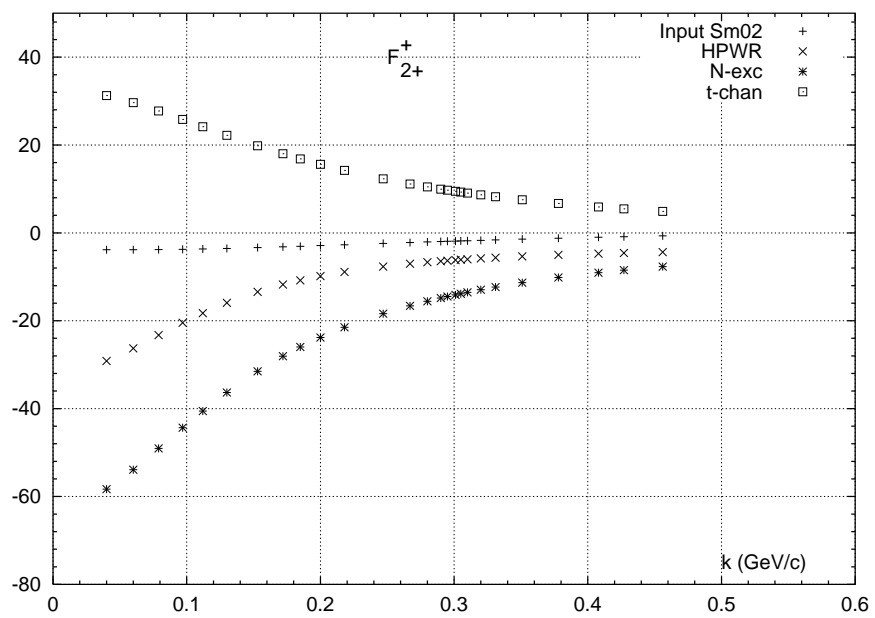

Figure 3: Comparizon of $\mathrm{F}_{2+}^{+}$from HPWR to input from $\mathrm{Sm02}$.

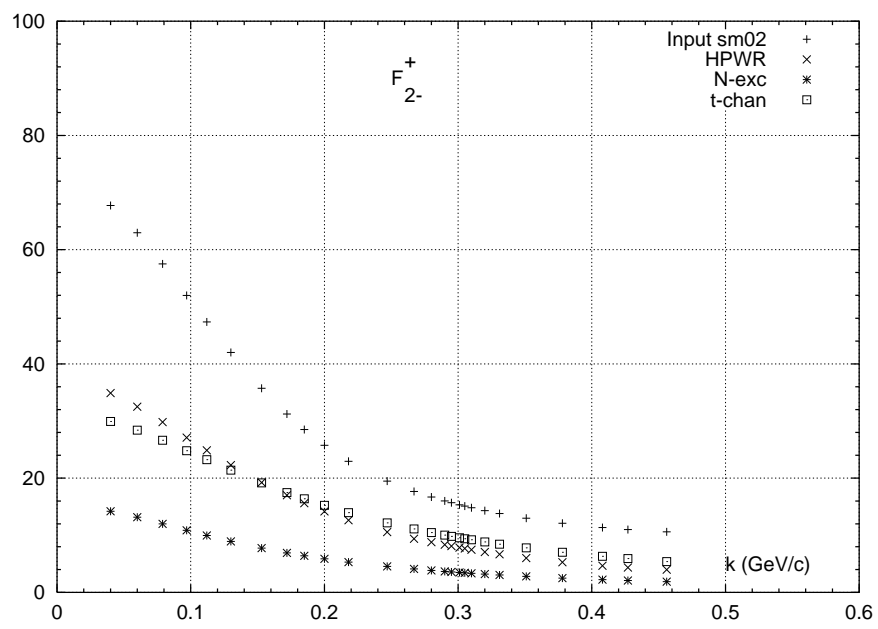

Figure 4: Comparizon of $\mathrm{F}_{2-}^{+}$from HPWR to input from Sm02. 


\section{Conclusions}

D waves in the VPI/GW Sp00 and Sm02 partial wave solutions are not consistent with analyticity in the low energy region and are evidently wrong. Methods sensitive to the input $\mathrm{D}$ waves produce high values of the pion nucleon sigma term because of the low energy D waves from VPI/GW solutions. A high "experimental value" of the sigma term could be accepted as reliable only if partial waves from the input partial wave solution are consistent with analyticity.

I wish to thank Prof. G. Höhler for his continuous support and valuable discussions. This work was supported by DOE project DE-FG03-94ER40860

\section{References}

[1] G. Höhler, Landolt-Börnstein, Vol. I/9b2, Pion-nucleon Scattering, Springer 1983.

[2] R. Koch, and E. Pietarinen, Nucl. Phys. A336 331 (1980)

[3] J. Gasser, H. Leutwyler, M. Locher, and M.E. Sainio, Phys. Lett. B213, $85,(1988)$.

[4] J. Gasser, H. Leutwyler, and M.E. Sainio, Phys. Lett. B253, 252(1991).

[5] M.E. Sainio, $\pi N$ Newsletter 13, 144(1997).

[6] R. Koch, Nucl. Phys. A448 707 (1986).

[7] M.E. Sainio, Proc. from the Inst. for Nuclear Theory, Vol.11, 346, ed. A.M. Bernstein, J.L. Goity, Ulf-G. Meißner, World Scientific 2000.

[8] SAID $\pi N$ database, http://gwdac.phys.gwu.edu.

[9] M.G. Olsson, Phys. Lett. B428, 50 (2000).

[10] M.G. Olsson, and W.B. Kaufmann, will be published in $\pi N$ Newsletter 16 (2002).

[11] J. Stahov, will be published in $\pi N$ Newsletter 16 (2002).

[12] G. Hite, and F. Steiner, Nuovo Cim. 18A 237 (1973).

[13] J. Stahov, Thesis, University Zagreb, 1983, J. Stahov, in preparation.

[14] J. Stahov, in preparation.

[15] M. M. Pavan, R. A. Arndt, I. I. Strakovsky, and R. L. Workman, will be published in $\pi N$ Newsletter 16 (2002).

[16] J. Hamilton," Pion Nucleon Interactions" in High Energy Physics, ed. E. H. S. Burhop, Academic Press, New York, 1964. 